\title{
External Ventricular Drainage Infections: A Single-Centre Experience on 100 Cases
}

Spazzapan Peter ${ }^{1 *}$, Bošnjak Roman ${ }^{1}$ and Zaletel Marjan ${ }^{2}$

${ }^{1}$ Department of Neurosurgery, University Medical Centre, Ljubljana, Slovenia

${ }^{2}$ Department of Neurology, University Medical Centre, Ljubljana, Slovenia

\begin{abstract}
Objective: The aim of the study was to identify the frequency of EVD infection, the risk factors for infection, the effect of infection to final outcome and to VP shunt insertion rate.

Methods: We retrospectively analysed 100 patients with EVD admitted to the Neurological ICU in the period from January 2013 to December 2014.

Results: The median age of the patients was 60.8 years (range $18-86$ years), $53 \%$ were male and $47 \%$ were female. The underlying pathologies were: subarachnoid haemorrhage $53 / 100(53 \%)$, intracerebral haematoma $43 / 100$ $(43 \%)$, tumour $3 / 100(3 \%)$ and cerebral infarction $1 / 100(1 \%) .33 / 100(33 \%)$ received a conservative treatment, $40 / 100(40 \%)$ a endovascular treatment and 29/100 (29\%) a surgical treatment. 13/100 (13\%) of the patients had an EVD infection. Statistical analysis showed no significant correlation between infection and age, underlying pathology and surgical treatment. There was a significant relationship between infection and longer EVD duration, multiple EVD replacements and multiple burr-holes. We classified the outcome at 6 months using the modified Rankin scale. The patients were divided into two groups: bad outcome (scores $3,4,5,6)$ and good outcome (scores $0,1,2)$. There was a significant correlation between age and bad outcome, but not between infection and bad outcome. $12(13.7 \%)$ patients without infection and $2(15.3 \%)$ patients with infection needed a permanent VP shunt. This difference was not statistically significant.
\end{abstract}

Conclusion: Infection is a potentially dangerous complication of EVD. We found an infection rate of $13 \%$, which is comparable with other literature reports. We noticed no correlation between infection and age, primary pathology or type of treatment. An EVD infection, if recognized soon and adequately treated, did not significantly affect the final outcome and the VPD insertion rate.

Keywords: External ventricular drainage; Ventriculitis; Meningitis; Antibiotic therapy; VP drainage; Subarachnoid haemorrhage; Intracerebral haematoma

\section{Introduction}

EVD (external ventricular drainage) is a frequently used procedure in neurological and neurosurgical ICU (intensive care unit). It allows CSF (cerebrospinal fluid) drainage and therefore it helps to control ICP (intracranial pressure) rises in patients with acute hydrocephalus. The main causes of acute hydrocephalus are SAH (subarachnoid haemorrhage), ICH (intracerebral haematoma) and tumours. A frequent complication of this procedure is infection, which is reported in literature in the range of 5-20\% [1-4]. Infection of an EVD leads to removal of the device, likely to its reinsertion, to prolonged hospital stay, increased costs and to higher morbidity. The purpose of this retrospective study is to analyse the experience of a single centre with EVDs, to evaluate the risk factors and the frequency of EVD infections and the effect of infection on the final outcome.

\section{Methods}

We retrospectively analysed all EVDs inserted in the ICU of the Neurological Clinic of the University Medical Centre Ljubljana in the period from January 2013 to December 2014. Patient demographics, underlying diagnosis, eventual development of infection, need for exchange or repositioning of the EVD, duration of drainage, clinical outcome and need for permanent VP shunt were recorded. No traumatologic and no paediatric patients were included, since these cases are routinely referred to other ICU of the same hospital. All patients with a pre-existent CSF infection were also excluded. The data gathering was often characterized by missing values. Strict inclusion and exclusion criteria were used to reduce this bias and all included patients had complete data collected. Furthermore all data were collected by a single researcher to reduce any additional variability.

Placement of the EVD was always performed under sterile conditions in the operating theatre [5]. Bed-side insertion was performed when the transfer to the operating theatre was precluded by urgent conditions [6,7]. The providers who placed the EVD had different experience levels, ranging from residents to old consultants. No data were collected, to assess if there were any trends towards higher infection rates in providers who were less experienced, compared with those who were more experienced.

After hair shaving and preparation of the operative field with standard sterile technique, the burr-hole was made through a $2.5 \mathrm{~cm}$ skin incision. In the cases of symmetrically dilated ventricles the burr-hole was placed around the Kocher's point and the EVD was inserted in the right frontal horn. In cases of asymmetrical dilatation, the ventricular catheter was inserted in the largest ventricular compartment. In case

*Corresponding author: Spazzapan Peter, Department of Neurosurgery University Medical Centre Ljubljana, Zaloška 7, Ljubljana 1000, Slovenia, Tel: 0038641233 759; Fax: 00386152222 18; E-mail: spazzapanpeter@yahoo.it

Received July 26, 2016; Accepted August 29, 2016 ; Published August 31, 2016

Citation: Peter S, Roman B, Marjan Z (2016) External Ventricular Drainage Infections: A Single-Centre Experience on 100 Cases. J Neurol Neurophysiol 7: 392. doi:10.4172/2155-9562.1000392

Copyright: ( 2016 Peter S, et al. This is an open-access article distributed under the terms of the Creative Commons Attribution License, which permits unrestricted use, distribution, and reproduction in any medium, provided the original author and source are credited. 
of haematocephalus the catheter was inserted into the side of major bleeding. The catheter was always tunnelled for at least $5 \mathrm{~cm}$. Basic CSF drainage Integra systems, with plain non-impregnated catheters were used in all cases. All patients received antibiotic prophylaxis with Cefazolin prior to EVD insertion. Prophylactic antibiotics were not used continually throughout the term in which the drain was in place. Prophylactic catheter exchange was never performed. The CSF was sampled in a sterile fashion for biochemical and microbiological analysis three times a week for the entire duration of EVD, by withdrawing $5 \mathrm{ml}$ of CSF through the proximal 3-way stopcock.

Definition of infection (ventriculitis, meningitis) was related to a febrile patient, with a positive CSF culture obtained by aspiration from the proximal 3-way tap, associated with CSF pleocytosis ( $>15$ cells/ $\mathrm{mm}^{3}$ ), high protein level with low glucose and appropriate clinical signs and symptoms (fever, meningism, reduced level of consciousness, photophobia, phonophobia) $[8,9]$. The term contamination was used when the patient had one single CSF culture positive for a common skin pathogen in the absence of abnormal CSF findings [4]. Antibiotic therapy was not given for simple contamination.

The EVD was always removed and eventually replaced in case of malfunctioning, obstruction, wrong position detected by CT scan, or infection. Drain blockage was sometimes resolved by rinsing the EVD with $0.9 \% \mathrm{NaCl}$.

Statistical analysis was done by using SPSS commercial software. Student T-test was used to analyse the differences between independent variables. The relationships between variables were tested using logistic regression analysis. A statistical significant result was considered when $\mathrm{p}<0.05$.

\section{Results}

\section{General characteristics}

We analysed a total of 100 patients, who had at least one EVD inserted (Table 1). The median age of these patients was 60.8 years (with a range between 18 and 86 years), 53 (53\%) were male and 47 (47\%) were female. Primary underlying pathologies were SAH 53/100 (53\%), ICH 36/100 (36\%), posterior fossa ICH 7/100 (7\%), tumour 3/100 (3\%) and CVI 1/100 (1\%) (Figure 1). 44/53 (83\%) SAH were from ruptured aneurysms, $9 / 53(16.9 \%)$ were cryptogenic and were treated with conservative methods. Among those caused by ruptured aneurysms, $38 / 44$ (86.3\%) were coiled, 6/44 (13.6\%) were microsurgically clipped. Among ICH, including those in the posterior fossa, 20/43 (46.5\%) were surgically evacuated $(5 / 7$ (71.4\%) of those in posterior fossa). 23/43 (53.4\%) of the ICH were treated conservatively. All 3 tumours were treated with microsurgical excision. Overall 33/100 (33\%) patients received a conservative treatment, $40 / 100(40 \%)$ an endovascular treatment and 29/100 (29\%) a surgical treatment (haematoma evacuation, aneurysmal clipping and tumour excision) (Figure 2). $73 / 100(73 \%)$ of patients had no EVD replacement. $21 / 100(21 \%)$ had 1 replacement, 5/100 (5\%) had multiple replacements. 92/100 (92\%) had one single burr-hole through which one or more EVDs were inserted. $5 / 100(5 \%)$ had two, 3/100 (3\%) had 3 burr-holes. Median duration of EVD placement was 16.2 days (minimum 0 days, maximum 67 days).

\section{EVD infection results}

13/100 (13\%) of all patients developed an EVD infection (ventriculitis or meningitis) (Table 1). Median age of the patients in the infection group was 59.2 years, while median age of the patients in the no-infection group was 61.3 years. We found no statistically significant relationship between patient's age and EVD infection ( $\mathrm{p}=0.54)$. In the infection group 9 patients $(69.3 \%)$ were males, 4 (30.7\%) were female. In the no-infection group $43(49.4 \%)$ patients were males, 44 cases (48\%) were females. The median duration of EVD placement was 27 days in the infection group and 14.7 days in the no-infection group. This difference was statistically significant $(\mathrm{p}<0.01)$.

Primary pathology in the infection group was SAH in 5 cases (38.4\%), ICH in 7 cases (53.8\%), tumour in 1 case (7.7\%). Primary pathology in the no-infection group was SAH in 48 cases (55.1\%), ICH in 36 cases $(41.3 \%)$, tumour in 2 cases $(2.2 \%)$, CVI in 1 case $(1.1 \%)$

\begin{tabular}{|c|c|c|c|c|}
\hline & & $\begin{array}{c}\text { ALL } \\
\text { PATIENTS } \\
(n=100)\end{array}$ & $\begin{array}{c}\text { NO- } \\
\text { INFECTION } \\
(n=87)\end{array}$ & $\begin{array}{l}\text { INFECTION } \\
(n=13)\end{array}$ \\
\hline \multicolumn{2}{|l|}{ Median age } & 60.1 years & 61.3 years & 59.2 years \\
\hline \multirow{2}{*}{ Sex } & M & $53(53 \%)$ & $43(49.4 \%)$ & $9(69.3 \%)$ \\
\hline & $\mathbf{F}$ & $47(47 \%)$ & $44(48.2 \%)$ & $4(30.7 \%)$ \\
\hline \multirow{4}{*}{$\begin{array}{l}\text { Underlying } \\
\text { pathology }\end{array}$} & SAH & $53(53 \%)$ & $48(55.1 \%)$ & $5(38.4 \%)$ \\
\hline & $\mathrm{ICH}$ & $43(43 \%)$ & $36(41.3 \%)$ & $7(53.8 \%)$ \\
\hline & TUMOR & $3(3 \%)$ & $2(2.2 \%)$ & $1(7.7 \%)$ \\
\hline & CVI & $1(1 \%)$ & $1(1.1 \%)$ & $0(0 \%)$ \\
\hline \multirow{3}{*}{ Treatment } & SURGERY & $29(29 \%)$ & $25(28.7 \%)$ & $3(23 \%)$ \\
\hline & ENDOVASCULAR & $40(40 \%)$ & $34(39.1 \%)$ & $4(30.7 \%)$ \\
\hline & CONSERVATIVE & $33(33 \%)$ & $28(32.1 \%)$ & $6(46.1 \%)$ \\
\hline \multicolumn{2}{|c|}{ EVD replacement } & $27(27 \%)$ & $16(18.3 \%)$ & $13(84.5 \%)$ \\
\hline \multicolumn{2}{|c|}{ Two or more burr-holes } & $8(8 \%)$ & $3(3.4 \%)$ & $3(23 \%)$ \\
\hline \multicolumn{2}{|c|}{ EVD duration } & 16.2 days & 14.7 days & 27 days \\
\hline
\end{tabular}

Table 1: The table shows the demographic and clinical data of all the patients with $E V D$, of the patients in the no-infection group and of those in the infection group.

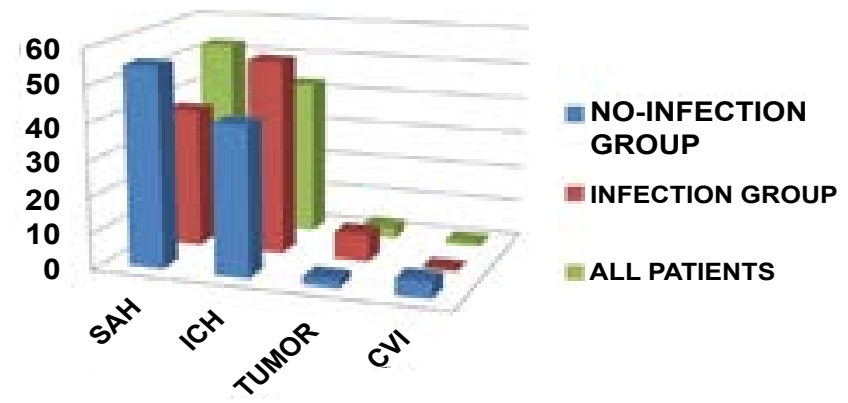

Figure 1: The figure shows the frequency of primary pathology among all patients. No significant correlation was found between infection and type of underlying pathology $(p=0.40)$

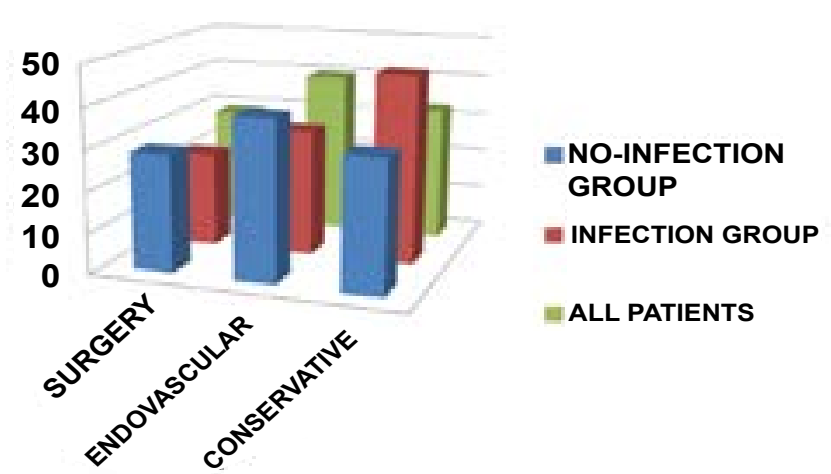

Figure 2: The figure shows the modality of treatment (surgical, endovascular conservative) in the no-infection and in the infection group. We found no significant correlation between infection and surgical treatment $(p=0.61)$. 
(Figure 1). We found no significant correlation between EVD infection and the two most common primary pathologies, $\mathrm{SAH}(\mathrm{p}=0.27)$ and $\mathrm{ICH}(\mathrm{p}=0.40)$.

Modality of treatment in the infection group was conservative in 6 cases $(46.1 \%)$, endovascular in 4 cases $(30 \%)$, surgical (evacuation of ICH, clipping, excision of tumour) in 3 cases (23\%). Modality of treatment in the no-infection group was conservative in 28 cases (32.1\%), endovascular in 34 cases $(39.1 \%)$ and surgical (evacuation of ICH, clipping, excision of tumour) in 25 cases (28.7\%) (Figure 2). We did not find any significant relationship between infection rate and surgical intervention $(\mathrm{p}=0.61)$.

In the infection group only 2 patients had one single EVD inserted (15.3\%). 8 cases $(61.5 \%)$ had one EVD replacement, while 3 patients $(23 \%)$ necessitated of multiple replacements. In 3 cases (23\%) the new EVD was inserted through a second burr-hole. In the no-infection group 71 patients $(81.6 \%)$ had one single EVD inserted. 13 cases (14.9\%) necessitated of one EVD replacement (because of obstruction, malfunctioning or wrong position). 3 cases (3.4\%) needed multiple (from 2 to 5) EVD exchanges. In 5 cases (5.7\%) the new EVD was inserted through a second burr-hole, in order to allow a better drainage of the ventricular system. We found a statistically significant relationship between EVD infection and multiple EVD replacements $(p<0.01)$, as well as between EVD infection and insertion through a second burr-hole $(\mathrm{p}=0.047)$.

Four cases of ventriculitis were caused by $S$. epidermidis and 2 cases were caused by E. cloacae. Other bacteria ( $S$. aureus, $P$. aeruginosa, $E$. coli, S. capitis, E. faecalis, S. haemoliticus, K. pneumoniae) were isolated from CSF each in one case.

\section{Outcome}

The outcome was measured using the modified Rankin score (mRS) which allows to classify patients in relation to their functional impairment (Figure 3). In the no-infection group the long term results were as follows: a score of 6 (death) was present in 25 cases (28.7\%). A score of 5 (very sick patient, who needs hospital admission and active supportive treatment) was reached by 16 patients (18.3\%). A score of 4 (patient who is unable to care for himself, who requires institutional or hospital care) was present in 15 cases (17.2\%). A score of 3 (patient who is unable to work, but able to live at home and care for most personal needs) was reached in 15 cases (17.2\%). A score of 2 (patient who is able to carry on normal activity and to work, without any special care needed) was reached by 9 patients (10.3\%). A score of 1 or 0 (normal patient, with very little or no complaints and no evidence of disease) was present in 7 cases (8\%) (Table 2).

In the infection group the results were as follows: 6 (death) in 2 cases (15.3\%), 5 in 2 cases (15.3\%), 4 in 2 cases (15.3\%), 3 in 5 cases (38.4\%), 2 in no case (0\%), 1 or 0 in 2 cases (15.3\%) (Table 2).

Based on the ability to care independently for themselves, to carry on normal daily activities and to work, we divided all outcomes in only 2 subgroups: one group of bad outcomes (Rankin scores 3, 4, 5, 6) and one group of fairly good outcomes (Rankin scores $0,1,2$ ) (Table 2 and Figure 4). In the no-infection group a bad outcome was present in 71 cases $(81.6 \%)$ and a good outcome in 16 cases $(18.4 \%)$. In the infection group, 11 cases $(78.6 \%)$ had a bad outcome and 3 cases $(21.4 \%)$ had a good outcome. We found no significant relationship between bad outcome and infection $(\mathrm{p}=0.65)$, but we found a significant relationship between bad outcome and age $(\mathrm{p}=0.026)$.

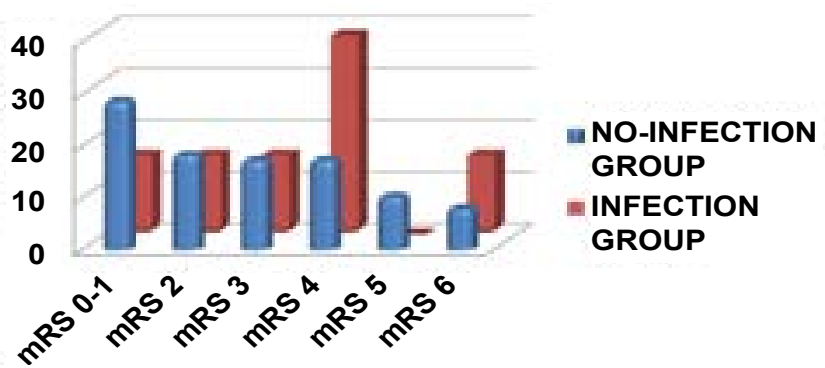

Figure 3: The figure shows the outcome of patients in the no-infection and in the infection group using the modified Rankin score.

\begin{tabular}{|c|c|c|}
\hline Modified Rankin scale & No-Infection $(\mathbf{n}=\mathbf{8 7})$ & Infection $(\mathbf{n = 1 3 )}$ \\
\hline 6 & $25(28.7 \%)$ & $2(15.3 \%)$ \\
\hline 5 & $16(18.3 \%)$ & $2(15.3 \%)$ \\
\hline 4 & $15(17.2 \%)$ & $2(15.3 \%)$ \\
\hline 3 & $15(17.2 \%)$ & $5(38.4 \%)$ \\
\hline 2 & $9(10.3 \%)$ & $0(0 \%)$ \\
\hline 1 or 0 & $7(8 \%)$ & $2(15.3 \%)$ \\
\hline Good outcome (scores 0, 1, 2) & $16(18.4 \%)$ & $3(21.4 \%)$ \\
\hline Bad outcome (scores 3, 4, & $71(81.6 \%)$ & $11(78.6 \%)$ \\
\hline 5,6$)$ & $12(13.7 \%)$ & $2(15.3 \%)$ \\
\hline
\end{tabular}

Table 2: The table shows the outcomes and the VP shunts rates of the patients in the no-infection and in the infection group.

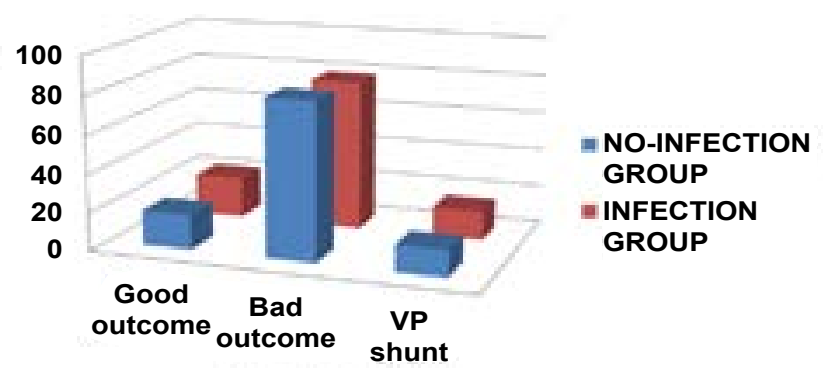

Figure 4: The figure shows the rate of good outcome (modified Rankin scores $0,1,2$ ) and bad outcome (modified Rankin scores $3,4,5,6$ ) and the rate of permanent VP shunt insertion in the no-infection and in the infection group.

Furthermore we performed a multivariate analysis, using the bad outcome as dependent variable and infection, EVD replacements, EVD duration and age as independent variables (Table 3). We found a significant negative association between bad outcome and EVD replacements $(\mathrm{p}=0.009$; Confidence interval $95 \%)$ and a positive significant association between bad outcome and age $(\mathrm{p}=0.014$ Confidence interval 95\%). The multivariate analysis showed neither a significant relationship between bad outcome and infection $(\mathrm{p}=0.205)$, nor between bad outcome and EVD duration $(\mathrm{p}=0.154)$.

Twelve patients in the no-infection group (13.7\%) needed a permanent VP shunt, to treat a chronic hydrocephalus. Two patients in the infection group (15.3\%) needed a permanent VP shunt (Figure 4). We found no statistically significant relationship between EVD infection and need for a permanent VP shunt $(\mathrm{p}=0.87)$.

\section{Discussion}

EVD is a life-saving procedure frequently used in ICU to treat acute hydrocephalus associated with $\mathrm{SAH}, \mathrm{ICH}$, tumours and other neurological urgencies. However, the high incidence of EVD infections 


\begin{tabular}{|l|l|l|l|l|l|}
\hline & B & Sig. & Exp (B) & \multicolumn{2}{|l|}{ 95\% C.I. for EXP (B) } \\
\cline { 5 - 7 } & & & & Lower & Upper \\
\hline Age & 0.054 & 0.014 & 1.056 & 1.011 & 1.102 \\
\hline EVD duration & 0.043 & 0.154 & 1.044 & 0.984 & 1.107 \\
\hline Infection & 1.258 & 0.205 & 3.517 & 0.503 & 24.611 \\
\hline EVD replacement & -1.454 & 0.009 & 0.234 & 0.078 & 0.698 \\
\hline Constant & -2.043 & 0.116 & 0.130 & & \\
\hline
\end{tabular}

Table 3: Multivariate analysis of the risk factors for bad outcome. Age and infection were significantly associated with bad outcome.

remains a major complication of this technique. The incidence of EVD infections is reported to be in the range between $4 \%$ and $23 \%$ in a large number of retrospective and prospective clinical studies [1,2,5,8,10-20]. A meta-analysis on 23 studies reported a cumulative rate of positive CSF cultures of $8.8 \%$ per patient, or $8.1 \%$ per EVD [4]. Nosocomial meningitis and ventriculitis represent a possible life-threatening condition, which may lead to a permanent adverse outcome of neurocritical patients.

The frequency of EVD infections in our cohort of patients was $13 \%$. This value is comparable with the infection rate in other previously published studies $[1,2,5,8,10-20]$.

Several factors have been detected, that can influence the EVD infection rate. These are EVD duration, EVD replacements, presence of a craniotomy, primary underlying pathology, antibiotic prophylaxis, CSF leakage, EVD tunneling and routine CSF sampling.

EVD duration longer than 5 or 10 days and EVD replacements were previously suggested to represent risk factors for infection $[4,8,15,21$ 26], though other authors have reported the opposite [14-16,27].

Holloway et al. $[11,24,26]$ demonstrated, that the risk of an EVD infection increased during the first 11 days, but after this period an infection became unlikely. Furthermore, it was demonstrated [28], that the median duration of EVD in patients with a CSF infection was actually longer than in patients without infection, but the prolongation of drainage time was a result of EVD infection and not vice versa [28].

Regarding EVD replacements, it has been reported [4], that the incidence of infection was $8 \%$ for every newly inserted EVD. Based on this it has been suggested, that infection may occur mainly at the moment of EVD placement and that multiple catheter exchanges may represent a risk factor for infection $[4,28]$.

Analysing the infection group in our cohort of patients, we recognized three statistically significant factors related to EVD infection: EVD duration, EVD replacements and multiple burr-holes. It is important to emphasise, that in cases of ventriculo-meningitis there was a need for a longer duration of EVD to obtain a good CSF clearance. Moreover an infection itself represented an indication for EVD replacement, if possible through a different burr-hole. We can therefore presume that in our study these three factors represented the consequences and not the causes of EVD infection.

Other recognized risk factors for an EVD infection are depressed cranial fractures [4,11], systemic infections [4] and craniotomies $[4,8,11]$. In our study the patients in the infection group received less frequently a surgical treatment $(23 \%)$, than patients in the no-infection group (28.7\%) and more frequently a conservative treatment (46.1\%) compared to the no-infection group (32.1\%). There was no significant relationship between EVD infection and craniotomy, suggesting that a major neurosurgical intervention may not represent a risk factor for EVD infection.

Regarding primary underlying pathology different studies showed an increased rate of infection in patients with SAH and intraventricular haemorrhage $[4,8,11,18]$. In our study we observed a slightly increased frequency of ICH cases in the infection group and an increased frequency of SAH in the no-infection group. Despite this, no significant correlation existed between infection and ICH or SAH, suggesting that in our cohort of patients the primary pathology had no influence on the development of an EVD infection.

Still controversial is the prophylactic use of antibiotics for EVD insertion. A meta-analysis published by Prabhu et al. [29] concluded, that the role of prophylactic antibiotics with EVD remains unclear. Other studies demonstrated, that procedural administration alone, or administration for the entire duration did not change the incidence of infections. A prospective randomised controlled study [30] suggested some advantage by using antibiotic for the entire duration of EVD, but noticed the disadvantage of selecting bacteria that caused more virulent infections. Regarding antibiotic impregnated catheters there are several studies demonstrating a significantly reduced bacterial adherence to these catheters and thus a significant reduction of CSF infections.

We did not use antibiotic impregnated catheters, but all patients received a dose of Cefazolin at the time of EVD insertion. We believe that this strategy represents a good protection against infection, without creating potentially insidious resistances to antibiotic treatment, which are frequently reported with the use of antibiotic impregnated catheters [31].

Another recognized risk factor for EVD infection is CSF leakage through the EVD insertion wound $[10,16,21,22,32]$. The leakage creates a direct connection between the intracranial and extracranial space, through which the cutaneous bacterial flora can migrate into the intracranial space. The absence of tunnelling of the EVD catheter is supposed to increase the rate of CSF leakage and therefore of EVD infections [3,23,33-35]. In fact the length of tunnelling has a close inverse correlation with the infection rate $[16,35]$ and this further suggests a connection between infection and the bacterial penetration through the EVD exiting point. Finally a major way of CSF infection is the routinely manipulation for CSF sampling $[19,24]$ and catheter rinsing $[1,4,5,8,27]$. For this reason some authors [24] suggested to limit CSF sampling to situations when there is clinical concern for CSF infection (fever, headache, change in mental status, nuchal rigidity, cranial nerve signs).

Data about the clinical status were collected 6 months after the treatment with EVD. We used the modified Rankin scale (mRS) to determine the functional outcome. The number of deaths in both groups was similar (28.7\% in the no-infection group and $15.3 \%$ in the infection group). The percentage of patients who received a score of 0 and 1 (normal patient with no or very little evidence of disease) was also comparable in the two groups (8\% in the no-infection and $15.3 \%$ in the infection group). The scores between these two extremes were also not much different in the two groups. In addition, we divided the outcome scores in two groups: one group of good outcome (Rankin score $0,1,2$ ) and one of bad outcome (Rankin score $3,4,5,6$ ). We found no significant relationship between bad outcome and infection, but we found a significant relationship between bad outcome and age. However these data should be interpreted carefully. Our sample size is relatively small and the conclusions are therefore weak. Despite 
this our data suggest that, beyond an eventual EVD infection, age and initial conditions of the patient are the main factors influencing the final outcome.

Similar results have been found also regarding the need for a permanent VP shunt. The VP shunt rate in our study was $13.7 \%$ in the no-infection group and $15.3 \%$ in the infection group. These results were similar to those of other studies, were the overall VP shunting rate was between $12 \%$ and $17.2 \%[36,37]$. There was no significant difference between the infection group and the no-infection group. We can assume that the main reason for the final insertion of a shunt is related to changes inside the ventricular system due to the primary pathology. Intraventricular scarring after a major bleeding is a wellknown risk factor for chronic hydrocephalus [38] and we can suppose that a ventricular infection adds only few more risks for the need of a permanent shunt.

Regarding the microbial agents in ventriculo-meningitis, the most frequently identified in literature $[4,39]$ are gram-positive cocci, consistent with normal skin flora such as Staphylococcus epidermidis (70\%), Staphylococcus aureus (10\%). Gram negative bacteria (Klebsiella spp., E. coli, Pseudomonas spp.) account for $15 \%$ of all infections, anaerobes and fungi such as Candida spp. $(<5 \%)$ are rare. Our results were comparable with these data, since $S$. epidermidis was the most common agent.

\section{Conclusion}

The results of our study indicate EVD duration, EVD replacements and replacements through multiple burr-holes as risk factors for EVD infection. Whether these three factors represent the direct causes or the consequences of an EVD infection is questionable, since an EVD infection requires by itself prolonged drainage duration, a catheter replacement and eventually the reinsertion through a different burrhole. Furthermore we found no statistically significant association between an EVD infection and a worse outcome, but a significant relationship between bad outcome and age. The multivariate analysis shows that bad outcome is significantly related to EVD replacements and age.

At first sight our results may suggest that an EVD infection, if treated early and correctly do not significantly affect the final functional outcome. These results shall however be read very carefully. Our sample size was relatively small; hence the conclusions are somewhat fragile. There is a worldwide intense interest in antibiotic prophylaxis in patients with EVD and in the use of antibiotic impregnated catheters. This is such an important topic because there is concern about the potential for infections resulting in a worsened outcome. Our evidence suggests that an EVD infection, if treated promptly and correctly can remain a contained event. The main factors influencing the final outcome remain patient's initial conditions and level of neurological damage.

\section{References}

1. Aucoin PJ, Kotilainen HR, Gantz NM, Davidson R, Kellogg P, et al. (1986) Intracranial pressure monitors. Epidemiologic study of risk factors and infections. Am J Med 80: 369-376.

2. Guyot LL, Dowling C, Diaz FG, Michael DB (1998) Cerebral monitoring devices: analysis of complications. Acta Neurochir Suppl 71: 47-49.

3. Friedman WA, Vries JK (1980) Percutaneous tunnel ventriculostomy. Summary of 100 procedures. J Neurosurg 53: 662-665

4. Lozier AP, Sciacca RR, Romagnoli MF, Connolly ES (2002) Ventriculostomyrelated infections: A critical review of the literature. Neurosurgery 51: 170-181.
5. Clark WC Muhlbauer MS, Lowrey R, Hartman M, Ray MW, et al. (1989) Complications of intracranial pressure monitoring in trauma patients. Neurosurgery 25: 20-24.

6. Kakarla UK, Kim LJ, Chang SW, Theodore N, Spetzler RF (2008) Safety and accuracy of bedside external ventricular drain placement. Neurosurgery 63 ONS162-166.

7. Ghajar JB (1985) A guide for ventricular catheter placement. Technical note. J Neurosurg 63: 985-986.

8. Mayhall CG, Archer NH, Lamb VA, Spadora AC, Baggett JW, et al. (1984) Ventriculostomy-related infections. A prospective epidemiologic study. N Eng J Med 310: 553-559.

9. Martínez R, Gaul C, Buchfelder M, Erbguth F, Tschaikowsky K (2002) Serum procalcitonin monitoring for differential diagnosis of ventriculitis in adult intensive care patients. Intensive Care Med 28: 208-210.

10. Bogdahn U, Lau W, Hassel W, Gunreben G, Mertens HG, et al. (1992) Continuous-pressure controlled, external ventricular drainage for treatment of acute hydrocephalus--evaluation of risk factors. Neurosurgery 31: 898-904.

11. Holloway KL, Barnes T, Choi S, Bullock R, Marshall LF, et al. (1996) Ventriculostomy infections: the effect of monitoring duration and catheter exchange in 584 patients. J Neurosurg 85: 419-424.

12. Kanter RK, Weiner LB, Patti AM, Robson LK (1985) Infectious complications and duration of intracranial pressure monitoring. Crit Care Med 13: 837-839.

13. Khan SH, Kureshi IU, Mulgrew T, Ho SY, Onyiuke HC (1998) Comparison of percutaneous ventriculostomies and intraparenchymal monitor: A retrospective evaluation of 156 patients. Acta Neurochir Suppl 71: 50-52.

14. Ohrström JK, Skou JK, Ejlertsen T, Kosteljanetz M (1989) Infected ventriculostomy: Bacteriology and treatment. Acta Neurochir (Wien) 100: 67-69.

15. Paramore CG, Turner DA (1994) Relative risks of ventriculostomy infection and morbidity. Acta Neurochir (Wien) 127: 79-84

16. Korinek AM, Reina M, Boch AL, Rivera AO, De Bels D, et al. (2005) Prevention of external ventricular drain--related ventriculitis. Acta Neurochir (Wien) 147: $39-45$.

17. Kim DK, Uttley D, Bell BA, Marsh HT, Moore AJ (1995) Comparison of rates of infection of two methods of emergency ventricular drainage. J Neurol Neurosurg Psychiatry 58: 444-446.

18. Stenager E, Gerner-Smidt P, Kock-Jensen C (1986) Ventriculostomy-related infections--an epidemiological study. Acta Neurochir (Wien) 83: 20-23.

19. Lucey MA, Myburgh JA (2003) Antibiotic prophylaxis for external ventricular drains in neurosurgical patients: An audit of compliance with a clinical management protocol. Crit Care Resusc 5: 182-185.

20. Pfisterer W, Mühlbauer M, Czech T, Reinprecht A (2003) Early diagnosis of external ventricular drainage infection: Results of a prospective study. J Neurol Neurosurg Psychiatry 74: 929-932.

21. Lyke KE, Obasanjo OO, Williams MA, O'Brien M, Chotani R, et al. (2001) Ventriculitis complicating use of intraventricular catheters in adult neurosurgical patients. Clin Infect Dis 33: 2028-2033.

22. Schade RP, Schinkel J, Roelandse FW, Geskus RB, Visser LG, et al. (2006) Lack of value of routine analysis of cerebrospinal fluid for prediction and diagnosis of external drainage-related bacterial meningitis. J Neurosurg 104: 101-108.

23. Khanna RK, Rosenblum ML, Rock JP, Malik GM (1995) Prolonged externa ventricular drainage with percutaneous long-tunnel ventriculostomies. J Neurosurg 83: 791-794.

24. Hoefnagel D, Dammers R, Ter Laak-Poort MP, Avezaat CJ (2008) Risk factors for infections related to external ventricular drainage. Acta Neurochir (Wien) 150: $209-214$

25. Arabi Y, Memish ZA, Balkhy HH, Francis C, Ferayan A, et al. (2005) Ventriculostomy-associated infections: Incidence and risk factors. Am J Infect Control 33: 137-143.

26. Park P, Garton HJ, Kocan MJ, Thompson BG (2004) Risk of infection with prolonged ventricular catheterization. Neurosurgery 55: 594-604.

27. Winfield JA, Rosenthal P, Kanter RK, Casella G (1993) Duration of intracranial pressure monitoring does not predict daily risk of infectious complications. Neurosurgery 33: 424-431. 
Citation: Peter S, Roman B, Marjan Z (2016) External Ventricular Drainage Infections: A Single-Centre Experience on 100 Cases. J Neurol Neurophysiol 7: 392. doi:10.4172/2155-9562.1000392

28. Hagel S, Bruns T, Pletz MW, Engel C, Kalff R, et al. (2014) External ventricular drain infections: Risk factors and outcome. Interdiscip Perspect Infect Dis.

29. Prabhu VC, Kaufman HH, Voelker JL, Aronoff SC, Niewiadomska-Bugaj M, et al. (1999) Prophylactic antibiotics with intracranial pressure monitors and external ventricular drains: A review of the evidence. Surg Neurol 52: 226-36.

30. Poon WS, Ng S, Wai S (1998) CSF antibiotic prophylaxis for neurosurgical patients with ventriculostomy: A randomised study. Acta Neurochir Suppl 71: 146-148.

31. Konstantelias AA, Vardakas KZ, Polyzos KA, Tansarli GS, Falagas ME (2015) Antimicrobial-impregnated and -coated shunt catheters for prevention of infections in patients with hydrocephalus: A systematic review and metaanalysis. J Neurosurg 122: 1096-1112.

32. Smith RW, Alksne JF (1976) Infections complicating the use of external ventriculostomy. J Neurosurg 44: 567-570.

33. Alleyne CH, Hassan M, Zabramski JM (2000) The efficacy and cost of prophylactic and perioprocedural antibiotics in patients with external ventricular drains. Neurosurgery 47: 1124-1127.
34. Saunders RL, Lyons TA (1979) External ventricular drainage. A technical note. Crit Care Med 7: 556-558.

35. Sandalcioglu IE, Stolke D (2003) Failure of regular external ventricular drain exchange to reduce CSF infection. J Neurol Neurosurg Psychiatry 74: 15981599.

36. Tso MK, Ibrahim GM, Macdonald RL (2015) Predictors of shunt-dependent hydrocephalus following aneurysmal subarachnoid hemorrhage. World Neurosurg 86: 226-232.

37. Lewis A, Irvine H, Ogilvy C, Kimberly WT (2015) Predictors for delayed ventriculoperitoneal shunt placement after external ventricular drain removal in patients with subarachnoid hemorrhage. Br J Neurosurg 29: 219-24.

38. Shooman D, Portess H, Sparrow O (2009) A review of the current treatment methods for posthaemorrhagic hydrocephalus of infants. Cerebrospinal Fluid Research 6: 1.

39. Dettenkofer M, Ebner W, Hans FJ, Forster D, Babikir R, et al. (1999) Nosocomial infections in a neurosurgery intensive care unit. Acta Neurochir (Wien) 141: 1303-1308. 